\title{
True vs Spurious Intrapartum Fetal Heart Rate Accelerations on the Cardiotocograph (CTG): An Urgent Need for Caution
}

\author{
Badriya Al Fahdii ${ }^{1}$ and Edwin Chandraharan ${ }^{2 *}$ \\ ${ }^{1}$ Oman Specialty Board, The Royal Hospital, Oman \\ ${ }^{2}$ Director, Global Academy of Medical Education \& Training, London
}

Submission: May 22, 2020; Published: June 04, 2020

*Corresponding author: Edwin Chandraharan, Director, Global Academy of Medical Education \& Training, London

\begin{abstract}
Transient increases in the fetal heart rate called "accelerations" on the cardiotocograph (CTG) trace during the intra partum period have been considered as 'hallmarks' of fetal wellbeing during labour. Several CTG guidelines have classified the fetal heart rate acceleration as a 'reassuring feature". The latest version of the Intra partum Fetal Heart Rate Monitoring Guidelines produced by the National Institute of Health and Care Excellence (NICE) in the UK states "the presence of fetal heart rate accelerations, even with reduced baseline variability, is generally a sign that the baby is healthy.
\end{abstract}

Keywords: Fetal heart; Reassuring feature; Cardio tocograph; Non-hypoxic causes

Abbreviations: CRG: Cardiotocograph; GBM: Gross Body Movements; CSF: Cerebrospinal Fluid; FSE: Fetal Scalp Electrode

\section{Introduction}

Transient increases in the fetal heart rate called "accelerations" on the cardiotocograph (CTG) trace during the intra partum period have been considered as 'hallmarks' of fetal wellbeing during labour. Several CTG guidelines have classified the fetal heart rate acceleration as a 'reassuring feature". The latest version of the Intra partum Fetal Heart Rate Monitoring Guidelines produced by the National Institute of Health and Care Excellence (NICE) in the UK states "the presence of fetal heart rate accelerations, even with reduced baseline variability, is generally a sign that the baby is healthy" [1]. However, it is very important to appreciate that only the "true" fetal heart rate accelerations reflect the integrity of the fetal somatic nervous system [2] however, there may be other "non-hypoxic causes" which may cause "spurious" accelerations, and these "spurious" accelerations may mask underlying fetal compromise. In addition, several pathological conditions such as intrauterine fetal convulsions, fetal hypotension and the pre terminal stage of fetal hypoxia with severe fetal metabolic acidosis may have transient increases in the fetal baseline heart rate "mimicking" accelerations. Therefore, it is very important to understand the definition of "true" accelerations which are a reassuring feature on the CTG trace, and differentiate these from "spurious" accelerations, which may occur in the presence of reduced baseline fetal heart rate variability, as a part of several underlying patho physiological processes that may affect the wellbeing of the fetus during labour.

\section{Definition of True Acceleration}

True fetal heart rate accelerations are defined as at least two episodes of abrupt and transient increase in the heart rate, with the amplitude of at least 15 BPM lasting for at least 15 seconds (but less than 10 minutes) during a 20-minute period [3]. It is important to appreciate that this abrupt and transient increase in the fetal heart rate should commence from the normal, stable baseline heart rate, which is appropriate for the gestational age of the given fetus, and then should return to the same stable baseline. It should not be part of a deceleration, and it should not occur from an abnormal or an unstable baseline (Figure 1). It has been shown that unlike the fetal breathing movements, the gross body movements (GBM) are controlled by the somatic nervous system responsible for fetal heart rate accelerations, and are not affected by maternal plasma glucose concentrations [4]. Moreover, with advancing gestational age, the association of FHR 


\section{Global Journal of Reproductive Medicine}

accelerations with GBM becomes stronger and approximately 90 per cent of accelerations occur with GBM [4]. In preterm fetuses, the movements were more frequent and but lasted for a shorter duration [4]. Due to the smaller muscle mass, both the amplitude and duration of accelerations in a preterm fetus would be much smaller (approximately 10 BPM lasting for 10 seconds) than at term [5]. The phenomenon of alternative epochs of active and quiet sleep, called "cycling" is an important parameter which denotes fetal well-being during the intrapartum period [6]. Considering that the median related time spent during the deep sleep is approximately $26 \%$ between 30 and 40 weeks of gestation [7], one should expect loss of accelerations during the periods of deep sleep. It has been shown that the median time spent during the quiet sleep is approximately 15.7 minutes (0-53.2) [7] and therefore, prolonged periods of loss of accelerations in term fetus during the antenatal period or during early labour should not be considered as normal. Therefore, one should be very cautious if the loss of accelerations continues in the presence of reduced baseline fetal heart rate variability. Presences of fetal heart rate accelerations that fulfil the criteria to be classified as "true" accelerations are associated with absence of acidosis at birth $[7,8]$. It has been shown that even in the presence of ongoing variable or late decelerations, if the baseline fetal heart rate variability remained normal, presence of acceleration is associated with absence of acidosis in approximately 97\% of foetuses [9]. Therefore, presence of "true" fetal heart rate accelerations should be considered as a very reassuring feature during labour. Although, historically it was believed that accelerations occur as a result of fetal body movements, it has been shown that neuromuscular blockade with gall amine abolished the fetal movements but not the accelerations [10]. Therefore, one should consider accelerations as centrally mediated through the somatic nervous system, and therefore, centrally acting medications can abolish accelerations. Animal studies have shown that the administration of adenosine directly into the cerebrospinal fluid (CSF), was associated with a progressive loss of fetal heart rate accelerations, most likely secondary to that reduction in the sympathetic outflow [11]. Similarly, the administration of cocaine can reduce the duration of fetal rapid eye movement (REM) sleep [12], and therefore fetal heart rate accelerations. "True" accelerations should have an abrupt onset from a normal, stable baseline which is appropriate for the gestational age of the given fetus in the presence of a reassuring variability, with amplitude of at least 15 BPM lasting for at least 15 seconds (and less than 10 minutes) in a term fetus. Any transient rise in the baseline heart rate which "mimics" true accelerations with reduced or abnormally increased amplitude or in the presence of reduced baseline variability should be considered as abnormal. A fetus has gross body movements during active sleep, and therefore, the baseline variability would be expected to be normal. During deep (quiet) sleep cycle with absence of eyeball movements and absence of gross body movements, which is characterised by reduced baseline fetal heart rate variability, one should not expect "true" accelerations, contrary to what is stated by the recent NICE Guidelines [1].

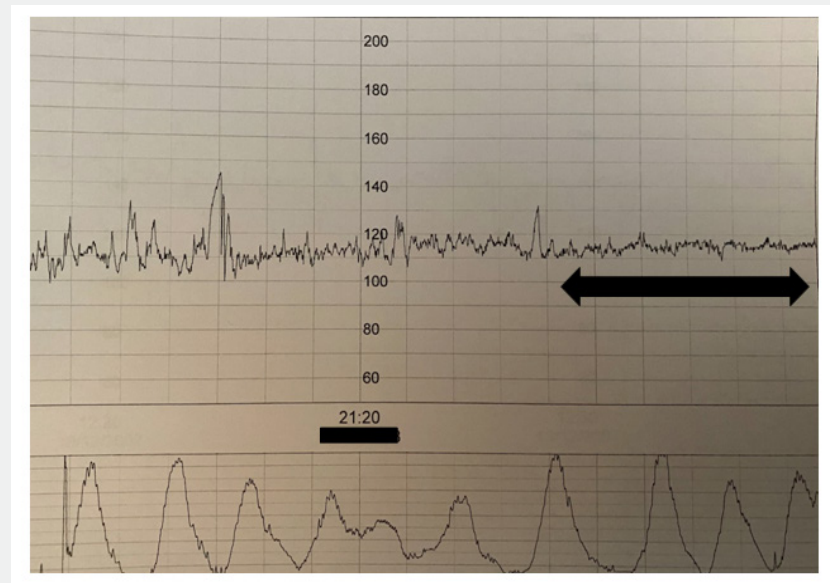

Figure 1: "True" fetal heart rate accelerations. Note that the accelerations are only present during the periods of normal variability, and not during the deep sleep phase (Double Arrow).

\section{Spurious Transient Increases in the Heart Rate Bimodal peaks}

\section{Mimicking the True Accelerations}

It is important to appreciate that anything that apparently rises about the baseline is not a true acceleration. Several hypoxic and non-hypoxic causes can masquerade as "True Accelerations" which may cause confusion during CTG interpretation leading to poor prenatal outcomes.
These usually occur during early labour, when uterine contractions selectively compress the "thin-walled" umbilical vein compared to the partial compression of the thick-walled muscular umbilical arteries. A sudden reduction in the blood flow secondary to the total compression of the umbilical vein would result in a transient fetal hypovolaemia and hypotension, 


\section{Global Journal of Reproductive Medicine}

leading to a compensatory sympathetic response to normalise the blood pressure. This may lead to a transient increase in the fetal heart rate, which is almost immediately followed by a sudden but a small drop in the fetal heart rate secondary to increased peripheral resistance as a result of the partial compression of the umbilical arteries. As the uterine contraction wanes. The umbilical arteries recoil back to normal, however, the umbilical vein remains compressed leading to another episode of fetal hypovolaemia and hypotension. This results in another sympathetic response resulting in a transient increase in the baseline heart rate, leading to a "Bimodal Peak" (Figure 2). These "Bimodal Peaks" coincide with ongoing uterine contractions, and have much larger amplitude as compared to normal fetal heart rate accelerations (Figure 2, Circle). They would eventually evolve having a longer declarative component as the umbilical arteries become more intensively compressed (Figure 3), ultimately culminating in "typical variable decelerations" (Figure 4) when the umbilical arteries are totally occluded by progressively increasing strength of uterine contractions. (Figure 3) Note the progression of the "Bimodal Peaks" with more pronounced drop in the heart rate in between the "shoulders" due to increasing occlusion of the umbilical arteries. These "Bimodal Peaks" secondary to early umbilical cord compression resulting in a selective, more intense compression of the "thin-walled" umbilical vein as compared to the "thick-walled" umbilical arteries (Figure 3) should not be misclassified as "True" Accelerations. The CTG trace should be carefully observed for the onset of "typical variable decelerations (Figure 4) and the fetal compensatory response should be assessed.

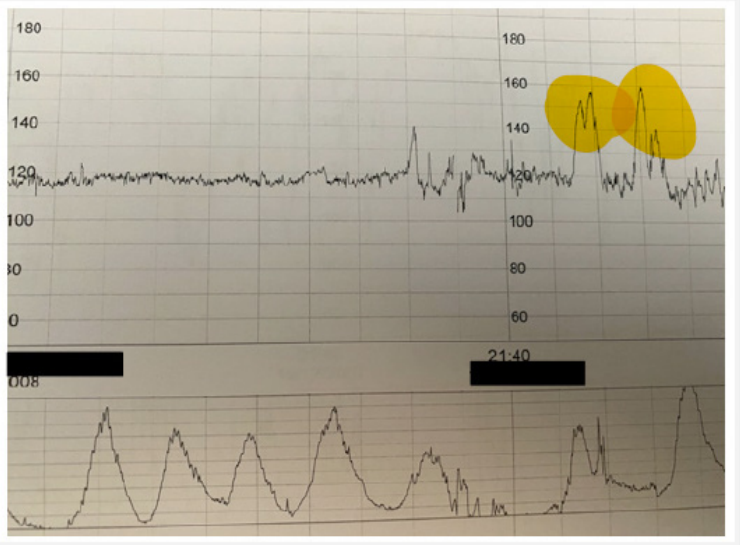

Figure 2: Note the "M-Shaped", "Bimodal Peaks" (Arrows) secondary to the selective, total compression of the umbilical vein, and only a partial compression of the umbilical arteries.

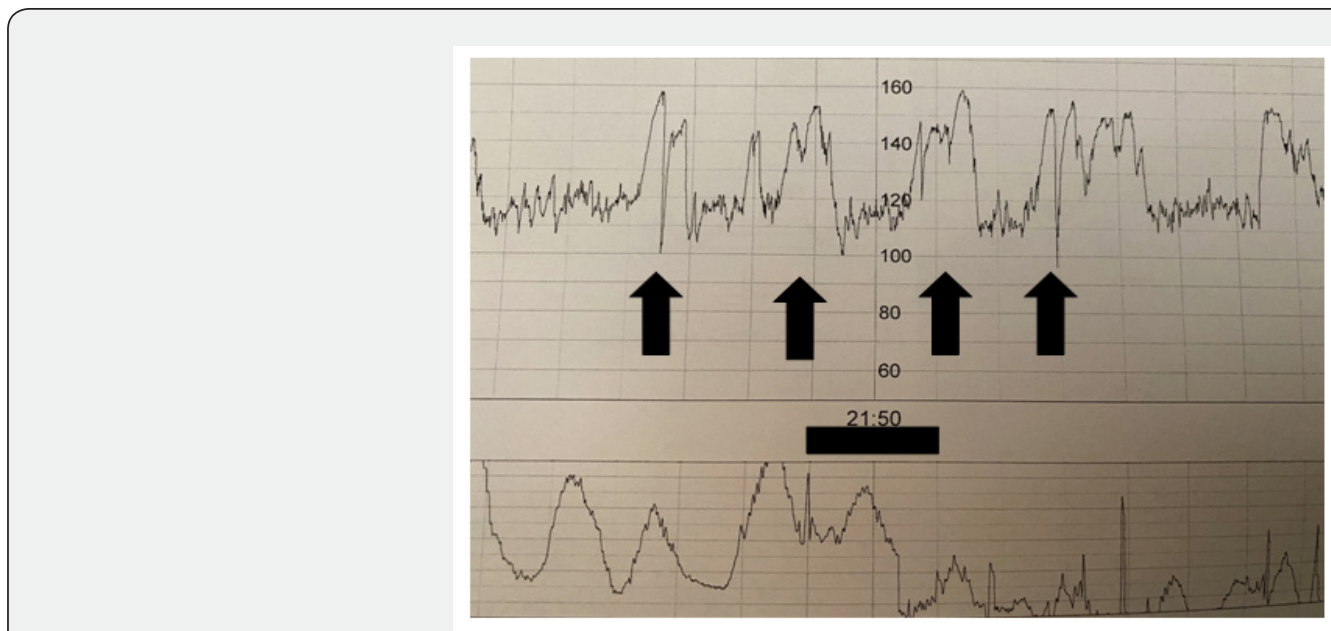

Figure 3: Note the progression of the "Bimodal Peaks" with more pronounced drop in the heart rate in between the "shoulders" due to increasing occlusion of the umbilical arteries.

\section{Shoulders of variable decelerations}

These are very common during human labour due to repetitive compression of the umbilical cord by ongoing uterine contractions. As the contraction begins, the thin-walled umbilical vein is selectively compressed leading to a sudden reduction in fetal blood flow resulting in fetal hypovolaemia and hypotension. An immediate sympathetic response to normalise 


\section{Global Journal of Reproductive Medicine}

the blood pressure would result in a transient increase in the heart rate giving rise to the first "shoulder" of a "typical" variable deceleration. As the uterine contraction intensifies, the umbilical arteries are firmly compressed leading to a sudden increase in the peripheral resistance and an abrupt increase in the fetal systemic blood pressure resulting in the stimulation of the baroreceptors $[11,12]$. This results in a sudden drop in the baseline fetal heart rate. As the uterine contraction wanes, the thick-walled muscular umbilical arteries recoil back resulting in a rapid recovery of the deceleration to the normal baseline. However, as the thin-walled umbilical vein remains compressed for longer period of time as compared to the umbilical arteries, that is a further reduction in the blood flow to the fetus even after the contraction. This results in another episode of fetal hypovolaemia and hypotension leading to a compensatory rise the baseline heart rate, resulting in another shoulder following the deceleration (Figure 4).

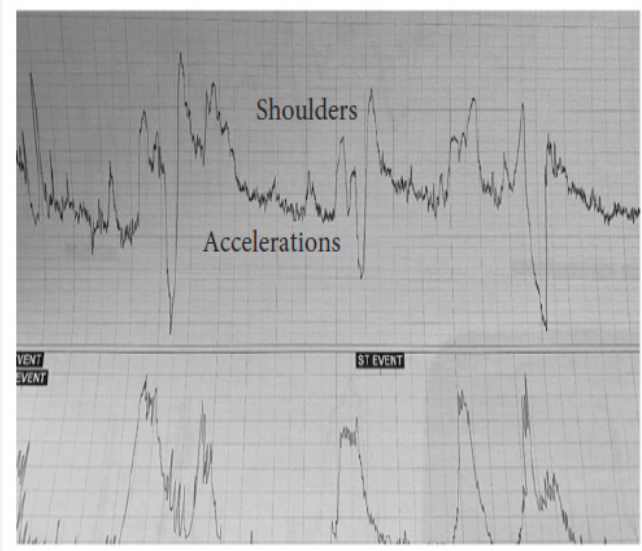

Figure 4: Note the "True Accelerations" (Arrows), and the "Shoulders" of typical variable decelerations (Circles).

\section{Fetal heart rate overshoots}

Overshoots appear as a highly exaggerated and transient, abrupt increases in the heart rate as a continuation of the ascending limb of the variable deceleration. It differs from the "shoulders" of the variable deceleration with regard to it's amplitude, duration and timing. Unlike the "uniform" increases in the heart rate both before and after a deceleration, with almost equal amplitude seen in a "typical" variable deceleration, an "overshoot" always occurs as a part of the "ascending limb" of the variable deceleration and has a wider duration with exaggerated amplitude (Figure 5). Fetal heart rate "overshoots" occur immediately following the peak of the underlying uterine contraction (Figure 5), as the fetus attempts to normalise the blood pressure by abruptly increasing the heart rate to compensate for ongoing, transient hypotension as a result of sustained and total compression of the umbilical cord. Experimental animal models (sheep) by Jenny Westgate et al. [13], have shown that whilst occluding the umbilical cord for one minute in every 5 minutes did not result in fetal heart rate "overshoots", if the umbilical cord is more repetitively compressed (one minute occlusions, for 4 times in 10 minutes), "overshoots" appeared on the CTG trace approximately after 30 minutes of commencement of umbilical cord occlusions. However, if the umbilical cord is more intensely included (sustained compression for two minutes, once in every 5 minutes), fetal heart rate "overshoots" appeared almost immediately [13]. It has been shown that these fetal heart rate "overshoots were associated underlying fetal hypotension and acidosis [13].Prolonged and repetitive umbilcial cord compression has been shown to be associated with fetal neuronal necrosis [14]. Therefore, these fetal heart rate "overshoots" observed on the CTG trace should not be misclassified as "accelerations" because they are neither normal nor reassuring. They represent an exaggerated fetal response to normalise ongoing episodes of transient fetal hypotension as a result of repetitive and sustained compression of the umbilical cord [15]. The intervening baseline fetal heart rate and the variability should be carefully scrutinised to assess oxygenation of the fetal central organs when "overshoots" are observed on the CTG trace. If oxytocin infusion is used to augment labour, the rate of infusion should be immediately reduced or stopped if fetal heart rate "overshoots" are repetitive or are associated with changes in the baseline fetal heart rate or variability [12]. Whilst fetal heart rate "overshoots" are very rare in spontaneous labour in the absence of strong and sustaineduteirne contractions, if they do occur, one needs to consider the possibility of severe olioghydramnios or anhydramnios +/- pre secence of thick meconium as the underlying cause for such repetitive and sustained compression of the umbilical cord [16]. During intermittent auscultation, if large amplitude accelerations are heard immediately after a uterine contraction, then, most likely these are fetal heart rate "overshoots" due to an unrecognised oligo or anhydramnios in a "low risk" labour. In such situations, the fetal heart rate should be auscultator throughout the next uterine contraction to detect a deceleration followed by an overshoot, so that continuous electronic fetal heart rate monitoring using a CTG can be commenced immediately to avoid poor prenatal outcomes. 


\section{The sloping cliff sign of a severe acidotic fetus}

It is important to appreciate that fetal heart rate decelerations are important protective reflex responses of the fetus with intact cardiovascular and neurological responses. Therefore, a healthy human fetus with intact compensatory responses would demonstrate an immediate, abrupt and deep drop in the baseline heart rate when presented with any hypoxic stress during labour. In contrast, the fetus who is hypo volaemic or acidotic for a prolonged period of time would lose such effective compensatory mechanisms, and the decelerations observed in such situations would be very shallow and less marked with a delayed recovery to the normal baseline fetal heart rate. The unsuccessful compensatory responses mounted by such an acidotic fetus "mimic" an acceleration, producing an "Sloping Cliff Sign" on the CTG trace, completely masking the underlying shallow deceleration (Figure 5). The slight increase in the baseline fetal heart rate immediately preceding a deceleration in response to the transient increase in the fetal blood volume with the commencement of uterine contraction and increased pressure within the placental venous sinuses, can be easily mistaken as an "acceleration". However, deeper understanding fetal patho physiology would help avoid such mistakes because as the fetus is acidotic, the baseline variability would be reduced in these cases (Figure 5). Moreover, these "transient and minor "increases in the baseline heart rate will be immediately followed by a shallow deceleration giving the appearance of a "Sloping Cliff". Therefore, irrespective of what has been stated erroneously by some guidelines on CTG interpretation ${ }^{1}$, transient increases in the fetal heart rate in the presence of a reduced baseline fetal heart rate variability should not be considered as "accelerations". This is because reduced baseline variability indicates that depression of the fetal central nervous system either due to fetal deep sleep, ongoing metabolic acidosis or other causes (medications, maternal acidosis, chorioamnionitis) which may depress the fetal central nervous system. In such cases, the fetal somatic nervous system which is present within the fetal brain would be depressed, leading to the absence of "true" fetal heart rate accelerations on the CTG trace.

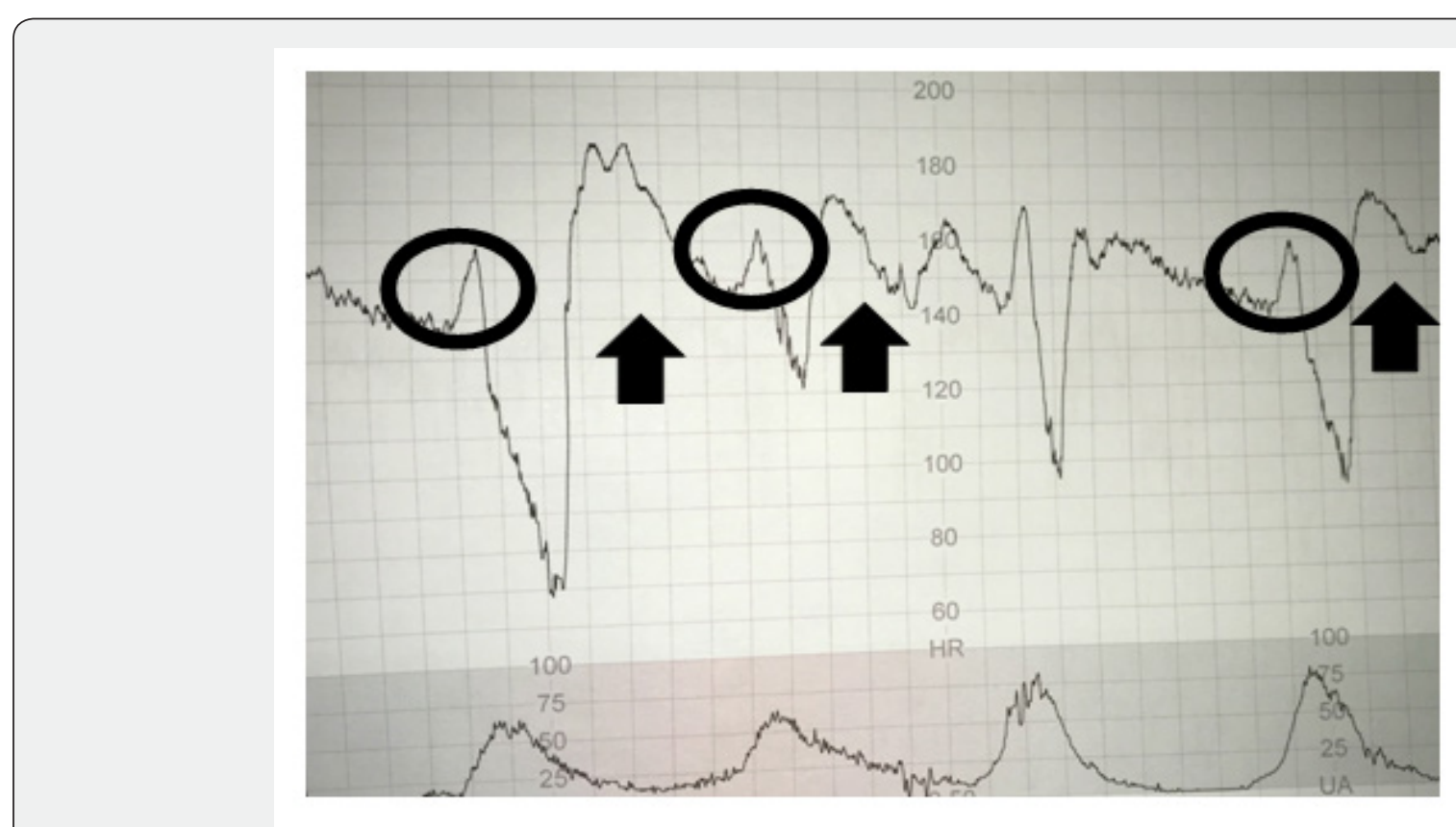

Figure 5: Note the transient and exaggerated amplitude of the increase in heart fetalrate as a part of the "ascending limb" of a variable decelerations(arrows) compared to the normal "shoulders" associated with the descending limb of decelerations (circles).

\section{Low amplitude flickers of intra-uterine convulsions}

Unlike the "true" fetal heart rate accelerations which occur with gross body movements (GBM) with an amplitude of at least 15 bpm lasting for at least 15 seconds in a term fetus, isolated and erratic fetal muscle activity secondary to intrauterine convulsions often produce "low amplitude flickers" on the CTG trace (Figure 6). The mother may complain of increased fetal moments, and in some cases an ultrasound scan may show the presence of such tonic and clonic movements of the fetal trunk and extremeities confirming ongoing intrauterine fetal convulsions [17].Intrauterine feel convulsions may occur due to an epileptogenic focus, in which case these convulsions would continue in the neonatal period [17], or as a terminal event in severe intrauterine fetal hypoxia and resultant metabolic acidosis, similar to neonatal convulsions 


\section{Global Journal of Reproductive Medicine}

observed in cases of severe peri partum hypoxia. A scientific review of the literature which analysed 22 cases of reported intrauterine convulsions suggests that apart from an "epileptigoneic focus" and intra partum asphyxia, other fetal disorders such as vitamin $\mathrm{B}_{6}$ deficiency, intrauterine fetal stroke, structural abnormalities in the fetal brain and neuromuscular disorders can also result in intrauterine fetal conversions [18]. In non hypoxic causes, the baseline variability would be maintained (Figure 6). In contrast, if the intrauterine fetal convulsions are due to an ongoing fetal metabolic acidosis or a disorder of the fetal brain which depress the autonomic nervous system, then the baseline variability would be reduced (Figure 7).

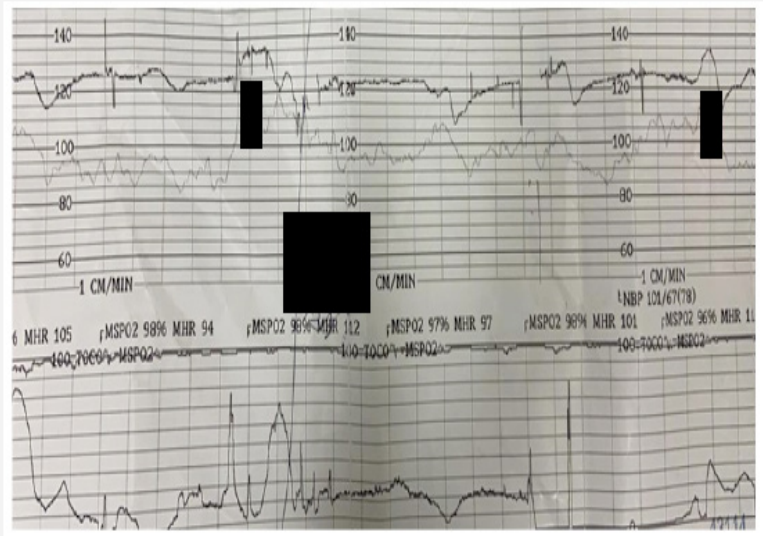

Figure 6: The slight increase in the baseline fetal heart rate immediately preceding a deceleration in response to the transient increase in the fetal blood volume with the commencement of uterine contraction and increased pressure within the placental venous sinuses, can be easily mistaken as an "acceleration".

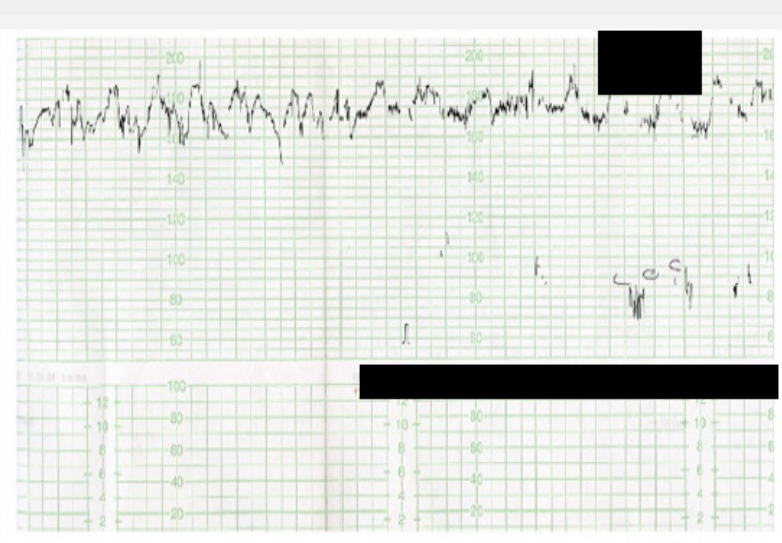

Figure 7: Note the repetitive low amplitude "flickers" with intervening normal baseline variability indicating a non-hypoxic cause for the ongoing intrauterine fetal convulsions.

\section{The "double mountain peak" sign: erroneous monitoring of maternal heart rate}

"True" Accelerations are hallmarks of a fetus in a nonhypoxic, non-acidotic state, which reflects the integrity and the oxygenation of the fetal somatic nervous system [12]. Therefore, it is imperative that the intelligent and highly evolved human fetus would expend his/her valuable resources (oxygen, glucose and other nutrients) on somatic muscle activity, only after ensuring adequate oxygenation of the central organs.It has been reported that the average oxygen saturation drops after a uterine contraction, with the greatest drop in oxygen saturation being reached 92 seconds after the peak of a contraction [19]. Moreover, it takes approximately 90 seconds for the reduced fetal oxygenation during a contraction to recover to its pre contraction levels [19]. Therefore, whilst it is usual for a fetus to show accelerations coinciding with uterine contractions in early labour [20] it is very unlikely to show repetitive accelerations during ongoing strong and sustained uterine contraction in late labour when fetal oxygen saturation would be reduced. This is because a fetus in late labour would attempt to normalise it's oxygenation saturation immediately after the contraction, and then may show 


\section{Global Journal of Reproductive Medicine}

accelerations secondary to the gross body movements during the inter- contraction interval when there is sufficient oxygen available the perfuse the central organs and to move the somatic muscles. Therefore, repetitive, transient and abrupt increases in the fetal heart rate coinciding with ongoing uterine contractions giving rise to the "Double Mountain Peak" sign on the CTG trace (Figure 8) should be considered as an abnormal feature in late labour. From a fetal patho physiological perspective, due to increasing frequency, duration and strength of uterine contractions in late labour and resultant repetitive and transient compression of the umbilical cord, the fetal head and/ or the reduction in the utero placental circulation, the normal reflex response of a healthy and intact fetus is a deceleration. On the contrary, due to the increasing pain, anxiety and increased blood volume during uterine contractions (i.e. as the pool of maternal blood per fusing the placenta is pushed back into the maternal circulation during uterine contractions), the normal physiological response of the mother is to increase the heart rate during contractions during the late stage of labour, especially during active maternal pushing. Therefore, accelerations coinciding with uterine contractions (Figure 8,9) giving the appearance of the "Double Mountain Peak" (i.e. the peak of acceleration sitting on the peak of contraction) should immediately arouse the suspicion of erroneous monitoring of the maternal heart rate as the fetal heart rate. Urgent action should be taken to confirm fetal heart rate recording by checking the maternal pulse, and the application of a fetal scalp electrode (FSE) if deemed appropriate. Sherman et al., [21] reported that the accelerations of the recorded maternal heart rate on the CTG trace have much larger amplitude as compared to expected accelerations of the fetal heart rate [21]. This should not be surprising to any practising clinician who understands human physiology because due to the large muscle mass, accelerations of the maternal heart rate during her expulsive efforts would have a very high amplitude as compare to fetal heart rate accelerations. It has been shown that accelerations coinciding with uterine contractions occur on the CTG trace during active maternal pushing in $12 \%$ of cases [22]. However, if an internal monitoring using the fetal scalp electrode (FSE) is employed, only $4 \%$ of CTG traces showed accelerations coinciding with uterine contractions during second stage of labour [22]. This illustrates the increasing likelihood of erroneously monitoring the maternal heart rate as the fetal heart rate on the CTG trace during second stage of labour with an external transducer, which is more likely to pick up signals from the pulsating maternal iliac blood vessels, as opposed to the signals from the fetal heart chambers which are located in a much deeper plane within the maternal birth passage.

Figure 8: In contrast, if the intrauterine fetal convulsions are due to an ongoing fetal metabolic acidosis or a disorder of the fetal brain which depress the autonomic nervous system, then the baseline variability would be reduced.

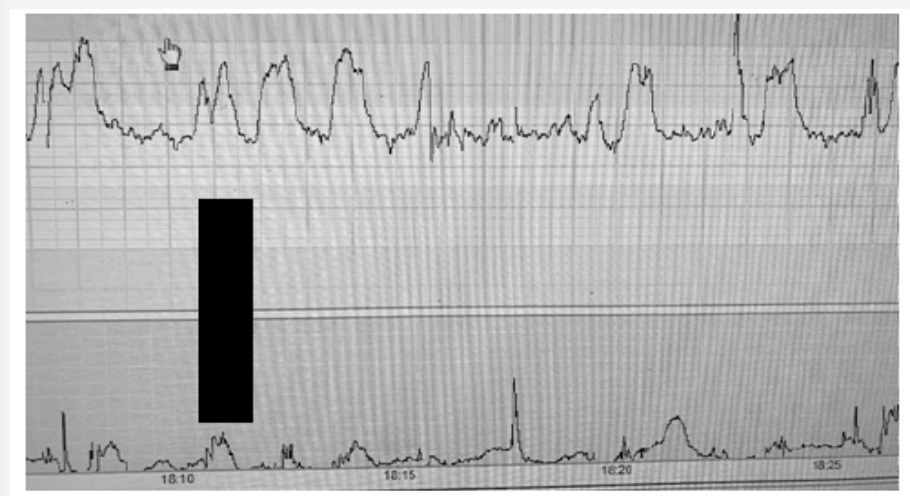

Figure 9: Note the large amplitude increases in the recorded heartrate coinciding with ongoing uterine contractions giving the appearance of a "Double Mountain Peak", resembling the recording on the tocograph. 


\section{Global Journal of Reproductive Medicine}

\section{Conclusion}

Anything that transiently and abruptly rises from a baseline heart rate should not be considered as a "true" fetal heart rate acceleration suggestive of an intact and well oxygenated fetal somatic nervous system, indicating the absence of an ongoing intra partum hypoxia and acidosis. "Spurious" increases in the fetal heart rate masquerading as "true" accelerations (Table 1) should always be considered by clinicians during intra partum period to avoid poor prenatal outcomes by carefully scrutinising all the features on the CTG trace. Contrary to what is erroneously stated by some guidelines on CTG interpretation [1], accelerations which occur in the presence of reduced baseline variability should be viewed with great caution. This is because normal fetal heart rate accelerations occur during the active phase of the fetal behavioural cycle, when there is no depression of the fetal central nervous system, and therefore, the variability should be normal during the periods of "true" accelerations. Disappearance of accelerations following the onset of decelerations may indicate ongoing gradually evolving hypoxia. High amplitude accelerations during labour, when the fetus is exposed to intra partum hypoxic stress are highly un physiological, and erroneous recording of the maternal heart rate and underlying repetitive and sustained umbilical cord compression (i.e. "overshoots") should be immediately excluded.

Table 1: Recognition \&Recommended Management of "Spurious" Accelerations.

\begin{tabular}{|c|c|c|}
\hline Spurious Acceleration & Underlying Mechanism & Recommended Action \\
\hline BimodalBimodal Peaks & $\begin{array}{l}\text { Selective compression of "thin-walled" umbilical } \\
\text { vein during early phases of umbilical cord } \\
\text { compression }\end{array}$ & $\begin{array}{l}\text { Careful observation as these often develop into } \\
\text { "typical variable declarations" with time }\end{array}$ \\
\hline $\begin{array}{l}\text { Shoulders" of "typical" variable decelera- } \\
\text { tions cal" variable decelerations }\end{array}$ & $\begin{array}{c}\text { Transient, "physiological" umbilical cord com- } \\
\text { pression }\end{array}$ & $\begin{array}{l}\text { Careful observation as these may develop in to } \\
\text { "atypical" or "complicated" variable decelerations. }\end{array}$ \\
\hline "Overshoot"Overshoots"s" & $\begin{array}{l}\text { Transient episodes of fetal hypotension second- } \\
\text { ary to repetitive and sustained compression of } \\
\text { the umbilical cord. }\end{array}$ & $\begin{array}{l}\text { Reduce ongoing stress (oxytocin), and exclude oli- } \\
\text { go/anhydramnios or thick meconium. Scrutinise } \\
\text { the CTG trace to assess oxygenation of fetal central } \\
\text { organs. }\end{array}$ \\
\hline The "Sloping Cliff Sign" & Severe fetal metabolic acidosis & $\begin{array}{l}\text { Immediate delivery after intrauterine resuscita- } \\
\text { tion (stop contractions) }\end{array}$ \\
\hline "Low Amplitude Flickers" & Intrauterine convulsions & $\begin{array}{l}\text { Intrauterine resuscitation (stop contractions) and } \\
\text { urgent delivery if the baseline FHR variability is } \\
\text { reduced. }\end{array}$ \\
\hline The "Double Mountain Peak" sign sign & $\begin{array}{l}\text { Erroneous recording of the high amplitude } \\
\text { maternal heart rate, giving the appearance of } \\
\text { another peak over the topography }\end{array}$ & $\begin{array}{l}\text { Check maternal pulse immediately and ensure } \\
\text { appropriate recording of the fetal heart rate }\end{array}$ \\
\hline
\end{tabular}

\section{References}

1. (2014) Intrapartum care for healthy women and babies.

2. Chandraharan E, Arulkumaran S (2007) Prevention of birth asphyxia: responding appropriately to cardiotocograph (CTG) traces. Best Pract Res Clin ObstetGynaecol 21(4): 609-624

3. Ayres de Campos D, Spong CY, Chandraharan E (2015) FIGO Intrapartum Fetal Monitoring Expert Consensus Panel. FIGO consensus guidelines on intrapartum fetal monitoring: Cardiotocography. Int J Gynaecol Obstet 131(1): 13-24.

4. Bocking AD (1989) Observations of biophysical activities in the normal fetus. Clin Perinatol 16(3): 583-594.
5. Afors K, Chandraharan E (2011) Use of continuous electronic fetal monitoring in a preterm fetus: clinical dilemmas and recommendations for practice. J Pregnancy 848794.

6. Preti M, Chandraharan E (2018) Importance offetal heart rate cycling during the interpretation of the cardiotocograph (CTG). Int J Gynecol and Reprod Sci 1(1): 10-12.

7. Suwanrath C, Suntharasaj T (2010) Sleep-wake cycles in normal fetuses. Arch Gynecol Obstet 281: 449-454.

8. Beard RW, Filshie GM, Knight CA (1971) The significance of the changes in the continuous fetal heart rate in the first stage of labour. J Obstet Gynaecol Br Commonw 78: 865-881. 
9. Williams KP, Galerneau F (2003) Intrapartum fetal heart rate patterns in the prediction of neonatal acidemia. Am J Obstet Gynecol 188(3): 820-823.

10. Clewlow F, Dawes GS (1985) The association between cardiac accelerations and movements in fetal sheep. J Dev Physiol 7(4): 281-287.

11. Egerman RS, Bissonnette JM, Hohimer AR (1993) The effects of centrally administered adenosine on fetal sheep heart rate accelerations. Am J Obstet Gynecol 169(4): 866-869.

12. Burchfield DJ, Peters AJ, Abrams RM, Phillips D (1995) Fetal behavioral state patterns during and after prolonged exposure to cocaine in sheep. Am J Obstet Gynecol 172(1): 1223-1228.

13. Jenny A Westgate, Laura Bennet, Harmen $H$ de Haan, Alistair J Gunn(2001) Fetal heart rate overshoot during repeated umbilical cord occlusion in sheep. Obstetrics \& Gynecology 97(3): 454-459.

14. De Haan HH, Gunn AJ, Williams CE, Gluckman PD (1997) Brief repeated umbilical cord occlusions cause sustained cytotoxic cerebral edema and focal infarcts in near-term fetal lambs. Pediatr Res 41(1): 96-104.

15. Pinas A, Chandraharan E (2016) Continuous cardiotocography during labour: Analysis, classification and management. Best Pract Res Clin ObstetGynaecol 30: 33-47.
16. McDonnell S, Chandraharan E (2015) Fetal heart rate interpretation in the second stage of labour: pearls and pitfalls. British journal of medicine and medical research 7: 957-970.

17. Jung E, Lee BY, Huh CY (2008) Prenatal diagnosis of fetal seizure: a case report. J Korean Med Sci 23(5): 906-908.

18. Usta I, Adra A, Nassar A (2007) Ultrasonographic diagnosis of fetal seizures: a case report and review of the literature. BJOG: An International Journal of Obstetrics \& Gynaecology 114: 1031-1033.

19. McNamara H, Johnson N (1995) The effect of uterine contractions on fetal oxygen saturation. BJOG: An International Journal of Obstetrics \& Gynaecology 102: 644-647.

20. Zimmer EZ, Divon MY, Vadasz A (1987) The relationship between uterine contractions, fetal movements and fetal heart rate patterns in the active phase of labor. Eur J Obstet Gynecol Reprod Biol 25(2): 89-95.

21. Sherman DJ, Frenkel E, Kurzweil Y, Padua A, Arieli S, et al. (2002) Characteristics of maternal heart rate patterns during labor and delivery. Obstet Gynecol 99: 542-547.

22. Nurani R, Chandraharan E, Lowe V, Ugwumadu A, Arulkumaran $S$ (2012) Misidentification of maternal heart rate as fetal on cardiotocography during the second stage of labor: the role of the fetal electrocardiograph. Acta Obstet Gynecol Scand 91(12): 1428-1432.

Your next submission with Juniper Publishers
will reach you the below assets
- Quality Editorial service
- Swift Peer Review
- Reprints availability
- E-prints Service
- Manuscript Podcast for convenient understanding
- Global attainment for your research
- Manuscript accessibility in different formats
( Pdf, E-pub, Full Text, Audio)
- Unceasing customer service
Track the below URL for one-step submission
https://juniperpublishers.com/online-submission.php

